\title{
The effects of dietary $n-3$ polyunsaturated fatty acids on neutrophils
}

\author{
Richard I. Sperling* \\ Department of Medicine, Harvard Medical School, Division of Rheumatology and Immunology, \\ Brigham \& Women's Hospital, Boston, MA 02115, USA
}

The present review will focus on the effects of dietary $n-3$ polyunsaturated fatty acids (PUFA) on the cellular function and leukotriene (LT) synthesis of human neutrophils. Dietary marine fish oils rich in $n-3$ PUFA have been shown to have an anti-inflammatory effect in patients with active rheumatoid arthritis (RA; Kremer et al. 1987, 1990; Sperling et al. 1987; Cleland et al. 1988; van der Tempel et al. 1990). This anti-inflammatory effect appears to be primarily due to the inhibitory effects of eicosapentaenoic acid (20:5n-3; EPA) on the formation of the lipid mediators of inflam- mation, which are derived predominantly from cellular phospholipids, and on the cellular function of inflammatory cells. The major lipid mediators of inflammation are the arachidonic acid (20:4n-6; AA) metabolites, the LT thromboxane $\mathrm{A}_{2}$ and the prostaglandins. Various inflammatory stimuli can activate cells to produce these mediators of inflammation. On cell activation (Fig. 1) phospholipase $\mathrm{A}_{2}$ (EC 3.1.1.4) is activated, which then catalyses the hydrolysis of PUFA from the 2-position of phospholipids yielding 2-lyso-phospholipids and the non-esterified PUFA. The

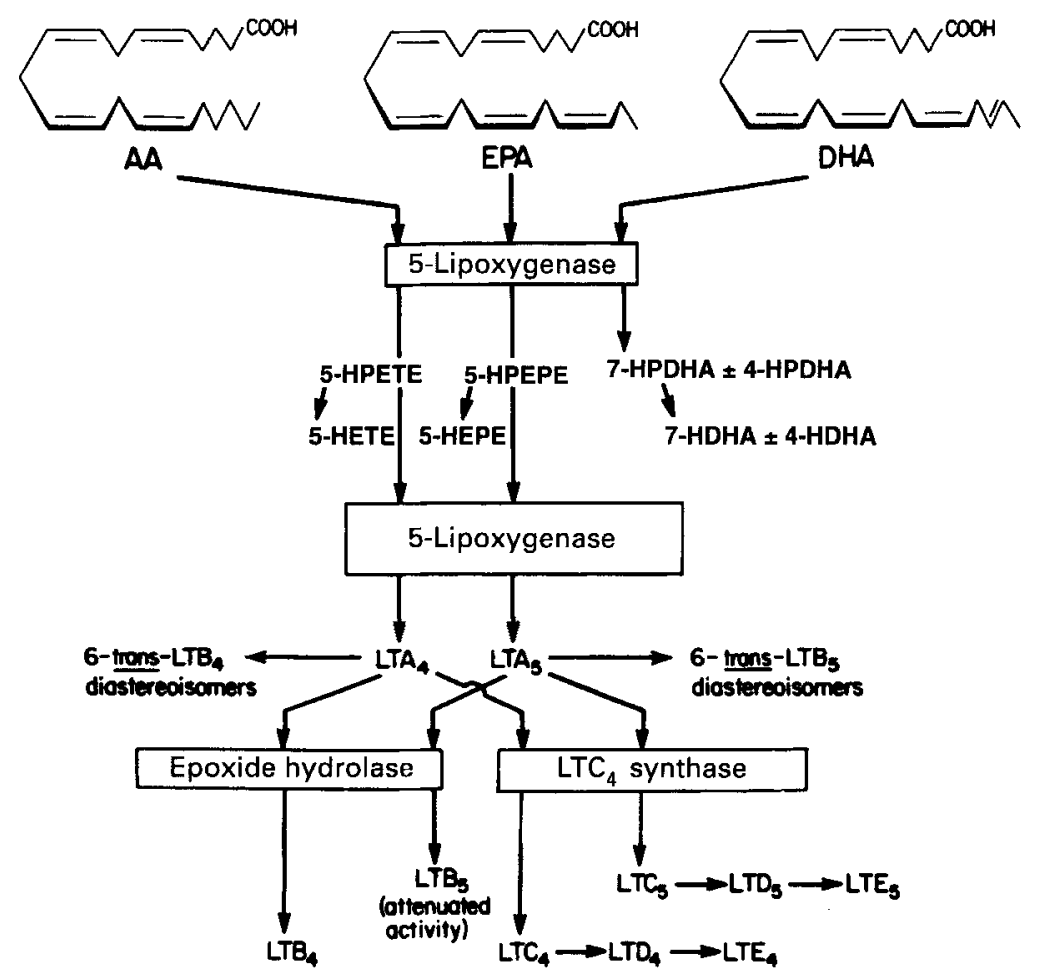

Fig. 1. Oxidative metabolism of polyunsaturated fatty acids arachidonic acid $(20: 4 n-6 ; A A)$, eicosapentaenoic acid (20:5n-3; EPA) and docosahexaenoic acid (22:6n-3; DHA) by the 5-lipoxygenase (EC 1.13.11.34) pathway. 5-HPETE, 5-S-hydroperoxyeicosatetraenoic acid; 5-HETE, 5-S-hydroxyeicosatetraenoic acid; 5-HPEPE, 5-S-hydroperoxyeicosapentaenoic acid; 5-HEPE, 5-S-hydroxyeicosapentaenoic acid; 7-HPDHA, 7-S-hydroperoxydocosahexaenoic acid; 4-HPDHA, 4-S-hydroperoxydocosahexaenoic acid; 7-HDHA, 7-S-hydroxydocosahexaenoic acid; 4-HDHA, 4-S-hydroxydocosahexaenoic acid; LT, leukotriene. (Adapted from Lee et al. 1985.)

\footnotetext{
Abbreviations: AA, arachidonic acid; DHA, docosahexaenoic acid; EPA, eicosapentaenoic acid; FMLP, N-formyl-methionyl-leucyl-phenylalanine; 5-HEPE, 5-S-hydroxy-trans-8,11,14-cis-eicosapentaenoic acid; 5-HETE, 5-S-hydroxy-trans-8,11,14-cis-eicosatetraenoic acid; 5-HPEPE, 5-S-hydroperoxy-6-trans8,11,14-cis-eicosapentaenoic acid; 5-HPETE, 5-S-hydroperoxy-6-trans-8,11,14-cis-eicosatetraenoic acid; HPDHA, hydroperoxy-docosahexaenoic acid; $\mathrm{IP}_{3}$, inositol-1,4,5-triphosphate; LT, leukotriene; PAF, platelet-activating factor; PLC, phospholipase C; PUFA, polyunsaturated fatty acid; RA, rheumatoid arthritis.
}

Corresponding author: Dr R. I. Sperling, fax +15088227975

*Present address: Health Associates of Taunton, Cutter Place, 152 Dean Street, Taunton, MA 02780, USA. 
non-esterified PUFA may serve as substrates for the cyclooxygenase ( $E C \quad 1.14 .99 .1)$ and 5-lipoxygenase $(E C$ 1.13.11.34) pathways. AA, the major substrate for both the 5-lipoxygenase and cyclooxygenase pathways in human subjects on a typical Western diet, is derived from $n$-6 PUFA of land-based animals and plants. EPA and docosahexaenoic acid $(22: 6 n-3 ;$ DHA) are the major fatty acids in marine organisms, found primarily in the form of esterified triacylglycerols. The well-established efficacy of the non-steroidal anti-inflammatory drugs in RA underscores the importance of the proinflammatory cyclooxygenase-pathway products in the pathophysiology of this disease. Studies of the synovial fluid from patients with active RA have demonstrated the presence of biologically-significant concentrations of $\mathrm{LTB}_{4} \quad(5 S, 12 R$-dihydroxy-6,14-cis-8,10-transeicosatetraenoic acid; Klickstein et al. 1980; Borenstein \& Vanderhoek, 1987) and 5-S-hydroxy-6-trans-8,11,14-ciseicosatetraenoic acid (5-HETE; Klickstein et al. 1980). $\mathrm{LTB}_{4}$ is not only one of the most potent chemotaxins for neutrophils and monocytes (Goetzl \& Pickett, 1980; Palmer et al. 1980), but also induces the release of lysosomal enzymes, the generation of highly-reactive reduced oxygen species, and also the aggregation of human neutrophil and monocytes (Yasaka et al. 1982). 5-HETE is a weak, partial agonist at the $\mathrm{LTB}_{4}$ receptor. These inflammatory mediators are believed to play a significant role in mediating the symptoms and joint destruction seen in RA (Robinson, 1988). The effects of the n-3 PUFA on leucocytes from healthy volunteers and patients with several defined inflammatory disorders has been evaluated. The clinical effects of dietary $n$-3 PUFA on disease activity in RA has been studied in a number of small clinical trials (Kremer et al. 1985, 1987, 1990; Sperling et al. 1987; Cleland et al. 1988; Tulleken et al. 1990; van der Tempel et al. 1990; Kjeldsen-Kragh et al. 1992; Nielsen et al. 1992; LG Darlington, personal communication).

\section{Pathway of the synthesis of leukotrienes}

Dietary PUFA, including the $n$-3 PUFA, are incorporated into membrane phospholipids, predominantly on the 2-position. On activation of phospholipase $A_{2}$, non-esterified fatty acids, primarily long-chain PUFA, and 2-lysophospholipids are released. Although AA and EPA are readily de-esterified from phospholipids, the hydrolysis of DHA-containing phospholipids by phospholipase $\mathrm{A}_{2}$ is disproportionately less.

AA is initially metabolized by 5 -lipoxygenase to its 5 $S$-hydroperoxy derivative, 5-S-hydroperoxy-6-trans-8,11, 14-cis-eicosatetraenoic acid (5-HPETE). Similarly, EPA and DHA are metabolized to their corresponding hydroperoxy derivatives, 5-S-hydroperoxy-6-trans-8,11,14-cis-eicosapentaenoic acid (5-HPEPE) and the corresponding 4- and 7-S-hydroperoxy-docosahexaenoic acids (HPDHA) respectively. DHA, however, is oxidatively metabolized by 5-lipoxygenase to a much lesser extent than either AA or EPA. These unstable hydroperoxy-fatty acid intermediates may, at least in part, be reduced to the corresponding alcohols known as 5-HETE, 5-HEPE, and 4- and 7-HDHA. This reduction is the final step in the metabolism of DHA by the 5-lipoxygenase pathway. 5-HPETE, derived from AA, and 5-HPEPE, derived from EPA, may also be metabolized further by the hydroperoxy-fatty acid dehydrase activity of 5-lipoxygenase to the respective 5,6-epoxides known as LTA $_{4}$ (5,6-trans-oxido-7,9-trans-11,14-cis-eicosatetraenoic acid), and the corresponding $\mathrm{LTA}_{5}$, derived from EPA (Rouzer et al. 1986).

The LTA epoxide LT are unstable in aqueous environments. The non-enzymic hydrolysis of AA-derived LTA mainly generates two minimally-bioactive diastereoisomeric dihydroxy-derivatives $(5 S, 12 R$ - and $5 S, 12 S$ - dihydroxy-6,8, 10-trans-14-cis-eicosatetraenoic acids) known as the 6-trans- $\mathrm{LTB}_{4}$ diastereoisomers. The epoxide hydrolase (EC 3.3.2.3), a cytosolic enzyme, is found in some human cells such as neutrophil (Rådmark et al. 1984), monocyte, erythrocyte and pulmonary alveolar macrophage and has also been detected in blood plasma (Fitzpatrick et al. 1983, 1984). The action of the LTA epoxide hydrolase on $\mathrm{LTA}_{4}$ generates the bioactive $\mathrm{LTB}_{4}$ (Fels et al. 1982; Godard et al. 1983; Williams et al. 1984; Borgeat \& Samuelsson, 1987). Analogously, the non-enzymic hydrolysis of LTA 5 generates the corresponding 6-trans- $\mathrm{LTB}_{5}$ diastereoisomers, whereas the action of the LTA epoxide hydrolase on LTA 5 generates LTB $_{5}$ (Lee et al. 1984a,b; Prescott et al. 1985; Strasser et al. 1985). The EPA-derived products, 5-HPEPE, 5-HEPE, $\mathrm{LTA}_{5}$, the 6-trans-LTB 5 diastereoisomers and $\mathrm{LTB}_{5}$, differ structurally from the corresponding AA-derived products only by the presence of an additional cis-double bond in the 17-position; however, $\mathrm{LTB}_{5}$, derived from EPA, possesses less than $10 \%$ of the bioactivity of $\mathrm{LTB}_{4}$ with respect to chemotaxis and lysosomal enzyme release (Lee et al. 1984a). In addition, recent evidence has indicated that the EPA-derived $\mathrm{LTB}_{5}$ can partially inhibit $\mathrm{LTB}_{4}$-mediated superoxide formation and chemotaxis (Kragballe et al. 1987). Thus, LTB $_{5}$ may actually act as a partial antagonist at the $\mathrm{LTB}_{4}$ receptor, in addition to its effects as a weak, partial agonist. $\mathrm{LTB}_{4}$ and $\mathrm{LTB}_{5}$ are oxidatively degraded by the neutrophil to their respective 20 -hydroxy derivatives by a unique cytochrome P450, referred to as $\mathrm{P}^{450_{\mathrm{LTB}}}$ (Shak \& Goldstein, 1985; Soberman et al. 1985). These minimallybioactive metabolites may be further oxidized by either of two pathways leading to the formation of 20-carboxyderivatives. However, 20 -hydroxy-LTB ${ }_{5}$ is metabolized to its 20 -carboxy derivative to only a minimal extent. In the monocyte, eosinophil and mast cell, $\mathrm{LTA}_{4}$ and $\mathrm{LTA}_{5}$ may be hydrolysed by $\mathrm{LTC}_{4}$ synthase to $\mathrm{LTC}_{4}$ and $\mathrm{LTC}_{5}$ respectively. Sequential cleavage of the glutathione side chain generates the biologically-active LT LTD $\mathrm{LT}_{4}$ and $\mathrm{LTE}_{4}$ from $\mathrm{LTC}_{4}$, and the bioactive $\mathrm{LTD}_{5}$ and $\mathrm{LTE}_{5}$ from $\mathrm{LTC}_{5}$ (Lee et al. 1985).

\section{Effects of $n-3$ polyunsaturated fatty acids on the human 5-lipoxygenase pathway}

\section{In vitro effects of $\mathrm{n}-3$ polyunsaturated fatty acids}

In the initial study of the in vitro effects of exogenous PUFA on 5-lipoxygenase-pathway product generation (Lee et al. $1984 b$ ), neutrophils were isolated from healthy donors who consumed their usual Western diet, and were activated $e x$ vivo with $10 \mu \mathrm{M}$-calcium ionophore A23187 for $5 \mathrm{~min}$ in the presence of exogenous quantities, $0-40 \mu \mathrm{g} \mathrm{AA}$, EPA and 
DHA/l. The formation of 5-lipoxygenase-pathway products was quantified by integrated optical density and/or radioimmunoassay after the resolution of products by reversephase HPLC. In the absence of exogenous fatty acids, the only products of the 5-lipoxygenase pathway which were detected were the stable products derived from endogenous AA in the neutrophil: 5-HETE, the 6-trans- $\mathrm{LTB}_{4}$ diastereoisomers and $\mathrm{LTB}_{4}$. The $\omega$-oxidation products of $\mathrm{LTB}_{4}$ were not clearly resolved by the HPLC programme and, therefore, were not quantified. The addition of concentrations of exogenous AA to the suspension of the neutrophils augmented the generation of each of these stable AA-derived 5-lipoxygenase products. In the presence of quantities of exogenous EPA, these same 5-lipoxygenase metabolites of AA, derived from cellular lipids, were demonstrated. In addition, small quantities of the analogous metabolites derived from exogenous EPA were detected. In the presence of exogenous EPA, there was a significant and selective reduction in the generation of $\mathrm{LTB}_{4}$, thus indicating inhibition of the LTA epoxide hydrolase enzyme. Ford- Hutchinson and colleagues (Nathaniel et al. 1985) have demonstrated that LTA $_{5}$ covalently binds to the LTA epoxide hydrolase, thereby inactivating it. In the presence of exogenous docosahexaenoic acid, the only DHA-derived 5-lipoxygenase products demonstrated were the 4- and 7-hydroxy derivatives, and exogenous DHA did not affect significantly the metabolism of endogenous AA by this pathway.

\section{The effects of dietary $\mathrm{n}-3$ polyunsaturated fatty acids on the 5-lipoxygenase pathway in healthy individuals}

Seven healthy, male volunteers daily supplemented their usual diet for 6 weeks with $18 \mathrm{~g}$ MaxEPA (R. P. Scherer, Troy, NY, USA) fish oil daily, providing $3.2 \mathrm{~g}$ EPA and $2.2 \mathrm{~g}$ DHA (Lee et al. 1985). Leucocytes were isolated and activated ex vivo with calcium ionophore in dose- and time-dependent protocols. 5-Lipoxygenase-pathway product generation was quantified by integrated optical density and/or radioimmunoassay after resolution of the products by reverse- phase HPLC. After 3 weeks of dietary fish oil, a 7 -fold increase in the EPA content of neutrophil cellular lipids was observed. This change persisted after 6 weeks of dietary fish oil consumption. The changes in neutrophil cellular lipid composition after 6 weeks of the dietary fish oil supplementation were associated with a $60 \%$ decrease in 5-HETE and 6-trans-LTB 4 generation, and a greater than 50 $\%$ decrease in $\mathrm{LTB}_{4}$ generation. Only small quantities of the EPA-derived 5-HEPE and $\mathrm{LTB}_{5}$ were detected after 6 weeks of dietary fish oil consumption. Despite comparable incorporation of EPA into neutrophil cellular lipids at the 3- and 6-week time points and comparable generation of EPAderived LTB $_{5}$ and 5-HEPE, suppression of 5-lipoxygenasepathway product formation was not observed after 3 weeks of dietary fish oil supplementation. The absence of an inhibitory effect of dietary $n-3$ PUFA on neutrophil 5-lipoxygenase-pathway product generation after the significant incorporation of $n-3$ PUFA into neutrophils which occurs after 3-4 weeks of dietary supplementation was also observed by Prescott et al. (1985) and Strasser et al. (1985). In their studies, after 3 and 4 weeks of dietary fish oil supplementation respectively, significant increases in neutrophil EPA contents and $\mathrm{LTB}_{5}$ formation were observed; however, suppression of $\mathrm{LTB}_{4}$ production was not demonstrated.

Terano et al. (1987) studied the effects of 4 weeks of dietary EPA ethyl ester and DHA ethyl ester, individually, on the function of neutrophils from healthy subjects. After 4 weeks of dietary EPA, small quantities of the EPA-derived 5-lipoxygenase-pathway products, 5-HEPE, the 6-trans$\mathrm{LTB}_{5}$ diastereoisomers and $\mathrm{LTB}_{5}$, were detected. $\mathrm{LTB}_{4}$ synthesis by calcium ionophore-activated neutrophils was inhibited by $34 \%$. No effects were observed on the synthesis of the other AA-derived 5-lipoxygenase-pathway products. In contrast, DHA did not affect neutrophil metabolism of AA by this pathway.

\section{The effects of dietary n-3 polyunsaturated fatty acids in patients with rheumatoid arthritis on leucocyte function and the 5-lipoxygenase pathway}

In the study by Sperling et al. (1987), twelve patients with active RA supplemented their diets for 6 weeks with $20 \mathrm{~g}$ MaxEPA fish oil daily. An 18-fold increase in EPA content and a concomitant $33 \%$ decline in AA content was observed after 6 weeks of the fish oil supplementation. Neutrophils were activated ex vivo with calcium ionophore in a doseand time-dependent protocol similar to that in the study of healthy individuals (Lee et al. 1985). A $50 \%$ inhibition in $\mathrm{LTB}_{4}$ generation and only small quantities of $\mathrm{LTB}_{5}$ were observed after 6 weeks of dietary fish oil supplementation. Although a $50 \%$ inhibition in $\mathrm{LTB}_{4}$ generation was observed both in healthy volunteers and the patients with RA, the generation of 5-HETE was inhibited by approximately $60 \%$ in the healthy volunteers after 6 weeks (Lee et al. 1985), but not in the RA patients (Sperling et al. 1987). Similar inhibition by dietary fish oil supplementation on $\mathrm{LTB}_{4}$ generation by neutrophils of patients with RA were observed by other investigators who employed different study designs (Kremer et al. 1987; Cleland et al. 1988).

\section{The effects of dietary $n-3$ polyunsaturated fatty acids on neutrophil function}

\section{The effects of dietary $\mathrm{n}-3$ polyunsaturated fatty acids on neutrophil function in healthy subjects}

In the study of healthy volunteers (Lee et al. 1985), neutrophil chemotaxis to $\mathrm{LTB}_{4}$ was assessed ex vivo in Boyden microchambers. The 6 weeks of dietary fish oil consumption resulted in a $71 \%$ decrease in the maximal neutrophil chemotactic responsiveness to $\mathrm{LTB}_{4}$, in addition to inhibiting 5-lipoxygenase-pathway product generation. Suppression of the $\mathrm{LTB}_{4}$-mediated enhancement of neutrophil adherence to endothelial cell monolayers (Hoover $e t a l$. 1984) was also observed (Lee et al. 1985). Neither of these changes were observed at the 3-week evaluation. These findings suggest that dietary fish oil supplementation might be expected to decrease both the margination and diapedesis of neutrophils into an inflammatory site in response to $\mathrm{LTB}_{4}$. Both the suppression of neutrophil chemotaxis and $\mathrm{LTB}_{4}{ }^{-}$ mediated enhancement of neutrophil-endothelial cell adhesion returned approximately to baseline levels 6 weeks 


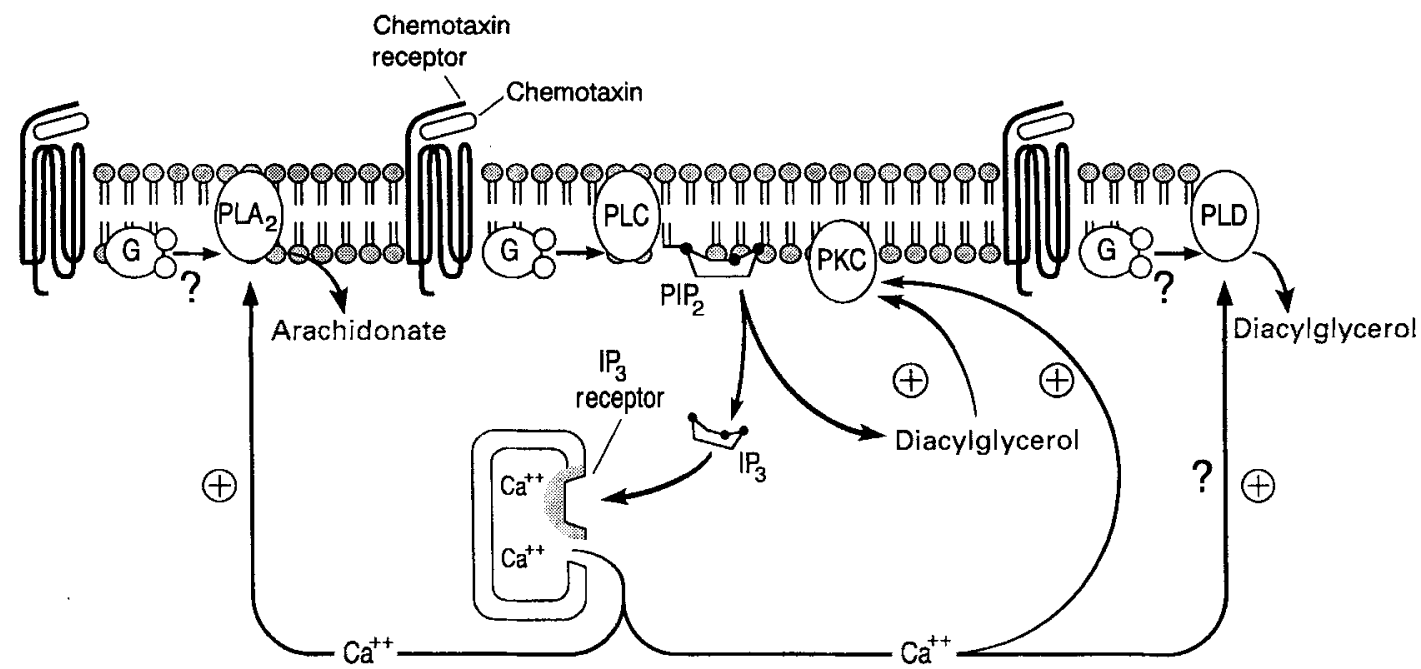

Fig. 2. Mechanisms of chemotaxin-induced activation of neutrophils. $G, G$-protein; $I P_{3}$, inositol-1,4,5-triphosphate; $P K C$, protein kinase $C$; $P L A_{2}$,

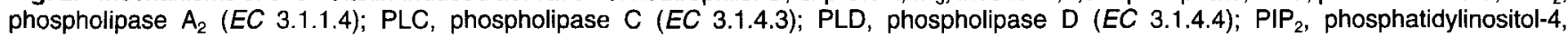
5-bisphosphate. $\oplus$, Activation.

after discontinuing the dietary fish oil, further supporting a causal relationship to the dietary modification. The suppression of neutrophil chemotaxis in the healthy volunteers is difficult to explain, as there is no clear link between chemotaxis and AA metabolism, as measured ex vivo, and no changes in neutrophil membrane fluidity were detected by fluorescence polarization in cells treated with the fluorescent probe, 1,6-diphenyl-1,3,5-hexatriene. The mechanism(s) by which dietary $n-3$ PUFA modulate transmembrane stimulation of neutrophils by chemotactic ligands were not addressed in this study.

In the study by Terano et al. (1987) of the effects of 4 weeks of dietary EPA ethyl ester and DHA ethyl ester, individually, on the function of neutrophils from healthy subjects, neutrophil chemotaxis to $\mathrm{LTB}_{4}$ and the chemotactic peptide $\mathrm{N}$-formyl-methionyl-leucyl-phenylalanine (FMLP) was assessed in Boyden microchambers. The authors observed a significant suppression of neutrophil chemotaxis to both agonists after 4 weeks of dietary EPA ethyl ester, supporting the findings of the previous study (Lee et al. 1985). DHA demonstrated a much less potent effect on neutrophil chemotaxis. The authors attributed the effect of DHA on neutrophil chemotactic responsiveness to a rise in the neutrophil cellular EPA content, observed after 4 weeks of DHA ethyl ester consumption (Terano et al. 1987).

Early events in the signal transduction after the binding of the neutrophil chemotactic ligands $\mathrm{LTB}_{4}$, plateletactivating factor (PAF) and FMLP to their respective receptors are believed to involve the activation of a G-protein which then activates an associated phosphatidylinositolselective phospholipase $C$ (EC 3.1.4.3; PLC; Fig. 2). The activated PLC hydrolyses phosphatidylinositol-4,5bisphosphate, resulting in the formation of inositol-1,4,5triphosphate $\left(\mathrm{IP}_{3}\right)$ and diacylglycerols. $\mathrm{IP}_{3}$ binds to specific receptors on the endoplasmic reticulum, resulting in the release of intracellular stores of $\mathrm{Ca}$ and activation of phospholipases $\mathrm{A}_{2}$ and $\mathrm{D}(E C$ 3.1.4.4). It is also possible that the receptor-ligand complex may activate phospholipase $\mathrm{D}$ and/or phospholipase $A_{2}$ directly through a $G$-protein. The diacylglycerols, in conjunction with the increased cytoplasmic $\mathrm{Ca}^{2+}$ concentration, activate protein kinase $\mathrm{C}$. The rise in intracellular $\mathrm{Ca}^{2+}$ concentration and the activity of protein kinase $C$ lead to activation of other specific cellular pathways, including the increased polarization of leucocytes, the assembly of elements of the cytoskeleton and cell contraction. It is possible that dietary fish oil supplementation affects any of these steps, resulting in inhibition of chemotaxis.

Sperling et al. (1993a) investigated the effects of 10 weeks of dietary $n-3$ PUFA supplementation with $20 \mathrm{~g}$ Super-EPA (Phenmacaps, Elizabeth, NJ, USA) daily, providing $9 \mathrm{~g}$ EPA and $5 \mathrm{~g}$ DHA in eight healthy volunteers, three men and five women, aged 25-59 years. Neutrophils and monocytes were isolated for biochemical investigations 4 weeks before starting the dietary supplementation and after 3 and 10 weeks of dietary $n-3$ PUFA supplementation. Increases in the EPA contents of all the major phospholipid classes and subclasses of neutrophils were observed after 3 and 10 weeks of dietary $n$-3 PUFA supplementation. There were no significant differences in the fatty acid contents of neutrophil phospholipids observed between the 3 and 10 week time points. Although the change in the EPA content of the phosphatidylinositol pool, of approximately $2 \mathrm{~mol} /$ $100 \mathrm{~mol}$, was much smaller than that observed in the other phospholipids, this change was nevertheless highly significant $(P<0.0001)$. The AA contents of the major phospholipid classes and subclasses of neutrophils, with the exception of the phosphatidylinositol pool, declined substantially after 3 and 10 weeks of dietary $n$-3 PUFA supplementation. The $\mathrm{LTB}_{4}$ and $\mathrm{PAF}$ receptor affinity and number of neutrophils did not change significantly with dietary $n-3$ PUFA supplementation.

The generation of inositol phosphates by neutrophils activated with $1 \mu \mathrm{M}-\mathrm{LTB}_{4}$ or $-\mathrm{PAF}$ for $15 \mathrm{~s}$ was quantified in $\left[{ }^{3} \mathrm{H}\right]$ inositol-labelled neutrophils before and after 3 and 10 weeks of dietary $n-3$ PUFA supplementation, by on-line $\beta$-scintillation counting after product resolution by anionexchange HPLC. The three and 10 weeks of dietary $n-3$ 
PUFA supplementation profoundly inhibited net formation of inositol monophosphate, diphosphate and $\mathrm{IP}_{3}$ in neutrophils stimulated with either agonist. Despite the minimal incorporation of EPA into the neutrophil phosphatidylinositol pool, formation of $\mathrm{IP}_{3}$ by both $\mathrm{LTB}_{4}$ and PAF-stimulated neutrophils correlated, by subject and time point, very significantly and negatively with the EPA contents of the respective phosphatidylinositol pools, as well as with other phospholipid pools. Dietary n-3 PUFA supplementation did not affect neutrophil diacylglycerol formation significantly. Under the conditions we employed, 2 min activation of cytochalasin B-treated neutrophils, most of the diacylglycerol formed would be derived from the phospholipase D pathway. Neutrophil chemotaxis to concentrations of $\mathrm{LTB}_{4}$ and PAF was assessed ex vivo in Boyden microchambers before and after 3 and 10 weeks of dietary $n-3$ PUFA supplementation. Neutrophil chemotaxis to each agonist was significantly inhibited after 3 weeks of dietary n-3 PUFA supplementation. A further significant inhibition of neutrophil chemotaxis to each of the ligands was observed after 10 weeks of dietary $n-3$ PUFA supplementation. Neutrophil chemotaxis to each of the ligands correlated significantly with $\mathrm{IP}_{3}$ formation, as determined for each subject at each time point, and also correlated negatively with the EPA contents of the phosphatidylinositol pools of the neutrophils of the respective subjects (Sperling et al. 1993a).

The inhibition of chemotaxin-stimulated $\mathrm{IP}_{3}$ formation, in the absence of an effect on the number of affinity of the respective chemotaxin receptors indicates that dietary $n-3$ PUFA supplementation inhibits the signal transduction pathway between the receptor and phospholipase $C$. This could occur at the level of the interaction of the receptor with the G-protein, the function of the G-protein itself, the activation of PLC by the G-protein, the intrinsic activity of PLC and/or at the level of PLC substrate availability, or an inhibitory effect on PLC of altered phosphatidylinositol species, the substrate of the enzyme.

The effects of dietary n-3 polyunsaturated fatty acids on the function of neutrophils in patients with rheumatoid arthritis

In the study by Sperling et al. (1987), in which twelve patients with active RA supplemented their diets for 6 weeks with $20 \mathrm{~g}$ of MaxEPA fish oil daily, the effects of dietary fish oil fatty acids on neutrophil chemotaxis to concentrations of $\mathrm{LTB}_{4}$ and FMLP were evaluated in Boyden microchambers. In the patients with RA, chemotaxis to both $\mathrm{LTB}_{4}$ and FMLP was suppressed in the pre-diet period as compared with that of healthy volunteers (Lee et al. 1985). After 6 weeks of dietary supplementation, neutrophil chemotaxis to $\mathrm{LTB}_{4}$ and FMLP increased towards normal. One possible explanation of these paradoxical findings is that in the pre-diet period the neutrophils of patients with RA were deactivated, perhaps due to in vivo $\mathrm{LTB}_{4}$ exposure; after the fish oil diet, there was less in vivo $\mathrm{LTB}_{4}$ exposure, and therefore less deactivation. This study, however, did not address the mechanism(s) of the effects of dietary $n-3$ PUFA supplementation on the chemotaxis of neutrophils from patients with RA.

Sperling et. al. (1993b) studied the mechanism of the differences in the baseline chemotaxin responsiveness of neutrophils from RA patients and previously studied healthy volunteers, and the effects of fish oil PUFA in sixteen patients with RA randomized to receive either $215 \mathrm{mg}$ fish oil PUFA or maize oil PUFA/kg per $d$ in a 10-week double-blind study. The methods used in this study are the same as the methods used in our parallel study in healthy volunteers (Sperling et al. 1993a). The maximal chemotactic response to $\mathrm{LTB}_{4}$ by neutrophils from RA patients was decreased by $84 \%$ compared with historical controls $(P<0.0001)$. This reflected a decrease in neutrophil $\mathrm{LTB}_{4}$ receptors and approximately $90 \%$ decrease in the $\mathrm{IP}_{3}$ signal generated by the $\mathrm{LTB}_{4}$ receptor in $\mathrm{LTB}_{4}$-stimulated neutrophils $(P=0.0001)$, as assessed by $\left[{ }^{3} \mathrm{H}\right] \mathrm{IP}_{3}$ formation in $\left[{ }^{3} \mathrm{H}\right]$ inositol-labelled polymorphonucleocytes stimulated by $\mathrm{LTB}_{4}$ and quantified by $\beta$-scintillation counting after resolution by HPLC. Similarly, formation of inositol mono- and diphosphates was decreased. In fish oil-PUFA-treated RA patients, an appreciable increase in $\mathrm{LTB}_{4}$ receptors was seen at week 10 , resulting in a small increase in $\mathrm{IP}_{3}$ formation and an increase in $\mathrm{LTB}_{4}$-mediated neutrophil chemotaxis. Significant changes in the neutrophils of maize oil-treated RA patients were not observed. The findings suggest that the decreased responsiveness to chemotaxins of neutrophils from RA patients is due to down-regulation of chemotaxin receptor number resulting in decreased signalling via chemotaxin receptors. Dietary fish oil PUFA partially reversed the down-regulation of the chemotaxin receptor of neutrophils of RA patients but had a lesser effect on chemotaxin receptor signalling and function, probably due to a post-receptor inhibition induced by fish oil PUFA, as was previously observed in healthy controls.

\section{The effects of dietary n-3 polyunsaturated fatty acids on disease activity in patients with rheumatoid arthritis}

Several small clinical studies (Kremer et al. 1985, 1987, 1990; Sperling et al. 1987; Cleland et al. 1988; Tulleken et al. 1990; van der Tempel et al. 1990; Astorga et al. 1991; Kjeldsen-Kragh et al. 1992; Nielsen et al. 1992; LG Darlington, personal communication) of the effects of low-dose dietary $n$-3 PUFA supplementation in patients with active RA each suggest modest improvement in disease activity. Larger, well-designed, multicentre trials are needed to determine the role of dietary $n-3$ PUFA supplementation in the treatment of RA, but have not been carried out to date due to the high cost of such trials and the lack of funding. Fortin et al. (1995) have objectively analysed the data from the studies already completed, using the techniques of meta-analysis and mega-analysis.

Studies were identified by a Medline literature search, checking references of the studies identified by the literature search, and by contacting reseachers in the field regarding any studies which they may be aware of, especially unpublished studies (Fortin et al. 1995). As part of the mega-analysis, eleven studies met our inclusion criteria. As part of the meta-analysis, for each publication, the methods and results sections were 'blinded' by the protocol of Chalmers et al. (1981). Quality scores for the methods and results were determined using the criteria of Chalmers $e t$ al. (1981) by two 'blinded', independent observers trained in quality scoring, but who were not otherwise involved in the study. Both the methods and results of each of these studies 
were rated of 'moderate quality' by the independent observers. The data were abstracted from the primary data of the original trials by the principal investigator of the original studies onto standardized data collection forms. Demographic data for the treatment and placebo groups were similar. A Chi-square test of homogeneity was performed for each outcome variable, and then rate differences and standard deviations for 3 months minus baseline were calculated between the treatment and the placebo groups for each outcome, and pooled rate differences with $95 \% \mathrm{Cl}$ were calculated using the DerSimonian \& Laird (1986) method. The meta-analysis revealed significant improvements in the tender/painful joint count and morning stiffness in the fish oil-supplemented group as compared with the control-oil group, after 3 months of treatment. Improvements observed in the other five outcome variables did not reach statistical significance. The results from the mega-analysis, as in the meta-analysis, show significant improvements in the tender or painful joint count and morning stiffness in the fish oilsupplemented group as compared with the control-oil group, after 3 months of treatment. Again, improvements in the other five outcome variables in the treatment group relative to the control group did not reach statistical significance. The results for the mega-analysis, as in the meta-analysis, show significant improvements in the tender or painful joint count and morning stiffness in the fish oil-supplemented group as compared with the control-oil group after 3 months of treatment. Again, improvements in the other five outcome variables in the treatment group relative to the control group did not reach statistical significance (Fortin et al. 1995).

\section{Effects of $n-6$ polyunsaturated fatty acids on the human 5-lipoxygenase pathway}

$\gamma$-Linolenic acid is an $n$-6 PUFA found in significant quantities in some land-based plants. In part, it is elongated to dihomo- $\gamma$-linolenic acid, a substrate for cyclooxygenase and 5-lipoxygenase. Ziboh \& Fletcher (1992) have shown that dietary supplementation with $\gamma$-linolenic acid-rich borage (Borago officinalis) oil suppresses $\mathrm{LTB}_{4}$ generation in calcium ionophore-activated neutrophils. In Dr Zurier's laboratory, similar findings were observed in monocytes (Pullman-Mooar et al. 1990), as well as evidence of suppressive effects on lymphocytes (Rossetti et al. 1997). This led to a few small clinical studies, spearheaded by Dr Robert Zurier, which suggested that plant oils rich in dihomo- $\gamma$-linolenic acid may improve disease activity in patients with active RA (Leventhal et al. 1993, 1994; Zurier et al. 1996).

\section{Summary and conclusions}

The studies of dietary fish oil supplementation in healthy volunteers demonstrate a significant increase in neutrophil EPA content, a concomitant reduction in neutrophil AA content, and suppression of neutrophil $\mathrm{LTB}_{4}$ synthesis by supplementation with dietary fish oil containing approximately 3-4 g EPA daily for a minimum of 4 weeks. Suppression of neutrophil chemotactic responsiveness to $\mathrm{LTB}_{4}$ and FMLP was observed after dietary $n$-3 PUFA supplementation at these levels. Dietary EPA is more active than
DHA in eliciting these effects in human neutrophils. Dietary $n$-3 PUFA supplementation inhibits neutrophil chemotaxis to these ligands through the inhibition of the signal transduction pathway between the receptor and phospholipase $\mathrm{C}$, as demonstrated by the inhibition of chemotaxin-stimulated $\mathrm{IP}_{3}$ formation, in the absence of an effect on the number or affinity of the respective chemotaxin receptors.

In patients with RA, dietary supplementation with $n-3$ PUFA resulted in decreased AA content of cellular lipids, with an augmented EPA content and decreased $\mathrm{LTB}_{4}$ generation by neutrophils. Dietary supplementation with $n-3$ PUFA also resulted in augmentation of depressed neutrophil chemotaxis to $\mathrm{LTB}_{4}$ and FMLP. Preliminary findings suggest that the decreased responsiveness to chemotaxins of neutrophils from RA patients is due to down-regulation of chemotaxin receptor number, resulting in decreased signalling via chemotaxin receptors. Dietary fish oil PUFA partially reversed the down-regulation of the chemotaxin receptor of neutrophils of RA patients, but had a lesser effect on chemotaxin receptor signalling and function, probably due to a post-receptor inhibition induced by fish oil PUFA, as was previously observed in healthy controls. Several small clinical trials have each suggested that dietary supplementation with $n$-3 PUFA resulted in modest improvements in disease activity. Meta-analysis of these studies confirms statistically significant improvements in tender joint count and morning stiffness after 3 months of dietary fish oil supplementation in patients with RA. Dietary supplementation with $\gamma$-linolenic acid-rich oils also inhibits neutrophil $\mathrm{LTB}_{4}$ formation, has other anti-inflammatory and immunosuppressive effects, and shows promise of therapeutic efficacy in RA.

\section{Acknowledgement}

This work was supported in part by Research Grants AR-01885 and AR-43518 from the National Institutes of Health.

\section{References}

Astorga GP, Cubillos AC, Masson LS \& Silva JJS (1991) Artritis rehumatoidea activa: Efecto de la suplementacion dietetica con aceites omega-3. Estudio doble-ciego controlado (Active rheumatoid arthritis: the effect of dietary supplementation with $0-3$ oils. A double-blind, controlled study). Revista Medica De Chile 119, 267-272.

Borenstein DG \& Vanderhoek JY (1987) Arthritis and Rheumatism 30, 565 Abstr.

Borgeat P \& Samuelsson B (1979) Arachidonic acid metabolism in polymorphonucleocytes: Effects of ionophore A23187. Proceedings of the National Academy of Sciences USA 76, 2148-2152.

Chalmers TC, Smith H Jr \& Blackburn B (1981) A method for assessing the quality of a randomized control trial. Controlled Clinical Trials 2, 31-49.

Cleland LG, French JK, Betts WH, Murphy GA \& Elliott MJ (1988) Clinical and biochemical effects of dietary fish oil supplements in rheumatoid arthritis. Journal of Rheumatology 15, 1471-1475.

DerSimonian R \& Laird N (1986) Meta-analysis in clinical trials. Controlled Clinical Trials 7, 177-188.

Fels AO, Pawlowski NA, Cramer EB, King TKC, Cohn ZA \& Scott WA (1982) Human alveolar macrophages produce LTB $_{4}$. 
Proceedings of the National Academy of Sciences USA $\mathbf{7 9}$, 7866-7870.

Fitzpatrick F, Haeggström J, Granström E \& Samuelsson B (1983) Metabolism of $\mathrm{LTA}_{4}$ by an enzyme in blood plasma: A possible leukotactic mechanism. Proceedings of the National Academy of Sciences USA 80, 5425-5429.

Fitzpatrick F, Liggett W, McGee J, Bunting S, Morton D \& Samuelsson B (1984) Metabolism of LTA 4 by human erythrocytes. A novel cellular source of leukotriene $\mathbf{B}_{4}$. Journal of Biological Chemistry 259, 11403-11407.

Fortin PF, Lew RA, Liang MH, Wright EA, Beckett LA, Chalmers TC \& Sperling RI (1995) Validation of a metaanalysis: The effects of fish oil in rheumatoid arthritis. Journal of Clinical Epidemiology 48, 1379-1390.

Godard P, Damon M, Michel FB, Corey EJ, Austen KF \& Lewis RA (1983) LTB $_{4}$ production from human alveolar macrophages. Clinical Research 31, 548A.

Goetzl EJ \& Pickett W (1980) The human PMN leukocyte chemotactic activity of complex hydroxyeicosatetraenoic acids (HETEs). Journal of Immunology 125, 1789-1791.

Hoover RL, Karnovsky MJ, Austen KF, Corey EJ \& Lewis RA (1984) LTB $_{4}$ action on endothelium mediates augmented neutrophil/endothelial adhesion. Proceedings of the National Academy of Sciences USA 81, 2191-2193.

Kjeldsen-Kragh J, Lund JA, Tove R, Finnanger B, Haaland K, Finstad R, Mikkelsen K \& Førre $\emptyset$ (1992) Dietary omega-3 fatty acid supplementation and naproxen treatment in patients with rheumatoid arthritis. Journal of Rheumatology 19, 1531-1536.

Klickstein LB, Shapleigh C \& Goetzl EJ (1980) Lipoxygenation of arachidonic acid as a source of polymorphonuclear leukocyte chemotactic factors in synovial fluid and tissue in rheumatoid arthritis and spondyloarthritis. Journal of Clinical Investigation 66, 1166-1170.

Kragballe K, Voorhees JJ \& Goetzl EJ (1987) Inhibition by leukotriene $\mathrm{B}_{5}$ of leukotriene $\mathrm{B}_{4}$-induced activation of human keratinocytes and neutrophils. Journal of Investigative Dermatology 88, 555-558.

Kremer JM, Bigauoette J, Michalek AV, Lininger L, Huyck C, Timchalk MA, Rynes RI, Zieminski J \& Bartholomew LE (1985) Effects of manipulation of dietary fatty acids on clinical manifestation of rheumatoid arthritis. Lancet i, 184-187.

Kremer JM, Jubiz W, Michalek A, Rynes RI, Bartholomew LE, Bigauoette J, Timchalk M, Beeler D \& Lininger L (1987) Fish oil fatty acid supplementation in active rheumatoid arthritis. Annals of Internal Medicine 106, 497-503.

Kremer JM, Lawrence DA, Jubiz W, DiGiacomo R, Rynes R, Bartholomew LE \& Sherman M (1990) Dietary fish oil and olive oil supplementation in patients with rheumatoid arthritis: Clinical and immunologic effects. Arthritis and Rheumatism 33, $810-820$.

Lee TH, Hoover RL, Williams ID, Sperling RI, Ravalese JR III, Spur BW, Robinson DR, Corey EJ, Lewis RA \& Austen KF (1985) Effect of dietary supplementation with eicosapentaenoic acid and docosahexaenoic acid on in vitro neutrophil and monocyte leukotriene generation and neutrophil function. New England Journal of Medicine 312, 1217-1223.

Lee TH, Mencia-Huerta J-M, Shih C, Corey EJ \& Austen KF (1984a) Characterization and biological properties of 5,12dihydroxy derivatives of EPA, including $\mathrm{LTB}_{5}$ and the double lipoxygenase product. Journal of Biological Chemistry 259, 2383-2389.

Lee TH, Mencia-Huerta J-M, Shih C, Corey EJ, Lewis RA \& Austen KF (1984b) Effects of exogenous arachidonic acid, eicosapentaenoic acid and docosahexaenoic acids on the generation of 5-lipoxygenase pathway products by ionophore activated human neutrophils. Journal of Clinical Investigation $\mathbf{7 4}$, 1922-1933.

Leventhal LJ, Boyce EG \& Zurier RB (1993) Treatment of rheumatoid arthritis with gammalinolenic acid. Annals of Internal Medicine 119, 867-873.

Leventhal LJ, Boyce EG \& Zurier RB (1994) Treatment of rheumatoid arthritis with blackcurrant seed oil. British Journal of Rheumatology 33, 847-852.

Nathaniel DJ, Evans JF, Leblanc Y, Leveille C, Fitzsimmons BJ \& Ford-Hutchinson AW (1985) LTA $_{5}$ is a substrate and an inhibitor of rat and human LTA $\mathrm{LA}_{4}$ hydrolase. Biochemical and Biophysical Research Communications 131, 827-835.

Nielsen GL, Faarvang KL, Thomsen BS, Teglbjaerg KL, Jensen LP, Hansen TM, Lerbang HH, Schmidt EB, Dyerberg J \& Ernst E (1992) The effects of dietary supplementation with $n-3$ polyunsaturated fatty acids in patients with rheumatoid arthritis: A randomized, double blind trial. European Journal of Clinical Investigation 22, 687-691.

Palmer RMJ, Stephaney RJ, Higgs GA \& Eakins KE (1980) Chemokinetic activity of arachidonic acid lipoxygenase products on leukocytes of different species. Prostaglandins 20, 411-418.

Prescott SM, Zimmerman GA \& Morrison AR (1985) The effects of a diet rich in fish oil on human neutrophils: Identification of leukotriene $\mathrm{B}_{5}$ as a metabolite. Prostaglandins 30, 209-227.

Pullman-Mooar S, Laposata M, Lem D, Holman RT, Leventhal LJ, DeMarco D \& Zurier RB (1990) Alteration of the cellular fatty acid profile and the production of eicosanoids in human monocytes by gamma-linolenic acid. Arthritis and Rheumatism 33, 1526-1533.

Rådmark O, Shimizu T, Jørnvall H \& Samuelsson B (1984) LTA $_{4}$ hydrolase in human leukocytes. Journal of Biological Chemistry 259, 12339-12345.

Robinson DR (1988) Rheumatoid arthritis. In Primer on the Rheumatic Diseases, 9th ed., pp. 24-30 [HR Schumacher, JH Klipple and DR Robinson, editors]. Atlanta, GA: Arthritis Foundation.

Rossetti RG, Seiler CM, DeLuca P, Laposata M \& Zurier RB (1997) Oral administration of unsaturated fatty acids: Effects on human peripheral blood $\mathrm{T}$ lymphocyte proliferation. Journal of Leukocyte Biology 62, 438-443.

Rouzer CA, Matsumoto T \& Samuelsson B (1986) Single protein from human leukocytes possesses 5-lipoxygenase and LTA $_{4}$ synthetase activities. Proceedings of the National Academy of Sciences USA 83, 857-861.

Shak S \& Goldstein IM (1985) Leukotriene $B_{4} \omega$-hydroxylase in human polymorphonuclear leukocytes. Journal of Clinical Investigation 76, 1218-1228.

Soberman RJ, Harper RW, Murphy RC \& Austen KF (1985) Identification and functional characterization of leukotriene $B_{4}$ hydroxylase in human polymorphonuclear leukocytes. Proceedings of the National Academy of Sciences USA 82, 2292-2295.

Sperling RI, Benincaso AI, Knoelle C, Austen KF \& Robinson DR (1993a) Dietary $\omega-3$ polyunsaturated fatty acids inhibit phosphoinositide formation and chemotaxis in neutrophils. Journal of Clinical Investigation 91, 651-660.

Sperling RI, Sohl P, Chen ZS, Lewis-Mannion M, Austen KF \& Robinson DR (1993b) Suppressed chemotactic responsiveness of PMN from patients with RA is due to downregulated receptor number and signaling. Arthritis and Rheumatism 36, S244 Abstr.

Sperling RI, Weinblatt M, Robin J-L, Ravalese JR III, Spur BW, Robinson DR, Corey EJ, Lewis RA \& Austen KF (1987) Effects of dietary supplementation with marine fish oil on leukocyte lipid mediator generation and function in rheumatoid arthritis. Arthritis and Rheumatism 30, 988-997.

Strasser T, Fischer S \& Weber PC (1985) Leukotriene $B_{5}$ is formed in human neutrophils after dietary supplementation with 
eicosapentaenoic acid. Proceedings of the National Academy of Sciences USA 82, 1540-1543.

Terano T, Seya A, Harai A, Saito H, Tamura Y \& Yoshida S (1987) Effect of oral administration of highly purified EPA and DHA on eicosanoid formation and neutrophil function in healthy subjects. In Proceedings of the AOCS Short Course on: Polyunsaturated Fatty Acids and Eicosanoids, pp. 133-138 [WEM Lands, editor]. Champaign, IL: American Oil Chemists' Society.

Tulleken JE, Limburg PC, Muskiet FAJ \& van Rijswijk MH (1990) Vitamin $E$ status during dietary fish oil supplementation in rheumatoid arthritis. Arthritis and Rheumatism 33, 1416-1419.

van der Temple H, Tulleken JE, Limburg PC, Muskiet FAJ \& van Rijswijk MH (1990) Effects of fish oil supplementation in rheumatoid arthritis. Annals of the Rheumatic Diseases 49 , $76-80$.
Williams JD, Czop JK \& Austen KF (1984) Release of leukotrienes by human monocytes on stimulation of their phagocytic receptor for particulate activators. Journal of Immunology 132, 3034-3040.

Yasaka T, Boxer LA \& Baehner RL (1982) Monocyte aggregation and superoxide anion release in response to formyl-methionylleucyl-phenylalanine (FMLP) and platelet-activating factor (PAF). Journal of Immunology 128, 1939-1944.

Ziboh VA \& Fletcher MP (1992) Dose-response effects of dietary $\gamma$-linolenic acid-enriched oils on human polymorphonuclearneutrophil biosynthesis of leukotriene $\mathbf{B}_{4}$. American Journal of Clinical Nutrition 55, 39-45.

Zurier RB, Rossetti RG, Jacobson EW, DeMarco DM, Liu NY, Temming JE, White BM \& Laposata M (1996) Gamma- linolenic acid treatment of rheumatoid arthritis: A randomized, placebocontrolled trial. Arthritis and Rheumatism 39, 1808-1817. 